\title{
ADVANCES IN GENOME INFORMATICS
}

\author{
XING-MING ZHAO*,§, YIXUE LI ${ }^{\dagger, \uparrow}$, MING LI $I^{\ddagger}, \|$ \\ and LUONAN CHEN ${ }^{\dagger}, * *$ \\ *Institute of Systems Biology, Shanghai University \\ Shanghai, 200444, P. R. China \\ ${ }^{\dagger}$ Key Laboratory of Systems Biology \\ Shanghai Institutes for Biological Sciences \\ Chinese Academy of Sciences, Shanghai 200032, P. R. China \\ ${ }^{\ddagger}$ David R. Cheriton School of Computer Science \\ University of Waterloo, Waterloo, \\ Ontario N2L 3G1, Canada \\ \$zhaoxingming@gmail.com \\ 『yxli@sibs.ac.cn \\ IImli@uwaterloo.ca \\ **Inchen@sibs.ac.cn
}

\section{Introduction}

This is the special issue devoted to the 21st International Conference on Genome Informatics (GIW-2010), the longest running international bioinformatics conference in the world, held during December 16-18, 2010, in Hangzhou, China. The 12 papers included in this special issue were selected from 75 submissions. These papers cover a broad range of topics including functional genomics, comparative genomics, biological network analysis, phenotype analysis, and so on. All the papers have gone through rigorous review procedure of the Journal of Bioinformatics and Computational Biology. The highlights of the selected papers are briefly introduced in the following.

\section{Molecular Annotation}

Molecular annotation is important for inferring biological functions of genes and properties of compounds. In this special issue, several papers presented new computational methods and new resources to infer gene functions and compound properties. For instance, Parasuram et al. ${ }^{1}$ presented a new pipeline for predicting the biochemical functions of un-characterized proteins with the assumption that proteins will share common functions if they have common functional sites. With the predicted functionally important residues based on 3D structures, the protein functions are expected to be predicted in a more reliable way than overall structure match. 
Comparative genomics has been considered as one major computational approach for predicting and analyzing gene functions for a long time. On this topic, Shih $e t ~ a l{ }^{2}$ developed a useful set of bioinformatics tools and an integrated database for marine and freshwater genomics, namely BiMFG. Even for an unknown marine or freshwater genome, it can be analyzed through BiMFG by conducting crossspecies comparison in sequences, motifs, and structures so that the gene functions can be inferred. Li et al. ${ }^{3}$ investigated the AFL genes that play a key role in seed development and maturation for plants by conducting genome-wide comparative analysis among various plant species. In particular, it was found that one AFL gene, i.e. ABI3, appears in all land plant genomes while some other genes, i.e. FUS3 and LEC2, appear only in specific genomes. The differences of these genes in evolutionary history were also demonstrated based on the study of comparative genomics.

Recently, network or graph theory emerges as a powerful tool for predicting protein functions. Yu et al. ${ }^{4}$ developed a new algorithm to predict the complexes by analyzing protein interaction network based on local density and random walks. With protein complexes found with local density as core, the attachment proteins were effectively detected by utilizing random walk algorithm. Compared with other methods, the proposed approach is able to find more biologically significant protein complexes.

Similar to molecular network, a compound can be represented as a graph with atoms as vertices and inter-atomic bonds as edges. With the compound structure represented as a graph, Brown et $a l^{5}$ proposed a new graph kernel method to predict compound properties by employing Support Vector Regression. Especially, the method was successfully used to predict the properties of a set of human vitamin $\mathrm{D}$ receptor ligands which have been found to have potential anti-cancer effects.

\section{Phenotype Analysis}

The understanding of mechanisms underlying phenotypes can help to design new drugs and combat diseases. In this issue, there are several new resources and methods contributed to the analysis of phenotypes.

Tohsato et $a l .{ }^{6}$ used phenotype microarray to elucidate the environmental dependency of genes, which can help to predict gene functions. In particular, a new statistical method was presented to analyze the phenotype data. Zhao et al. ${ }^{7}$ developed a new database for glucose-6-phosphate dehydrogenase (G6PD) deficiency, which is the most common hereditary enzymatic disorder of red blood cells in humans. Wei et al. ${ }^{8}$ analyzed the distribution of MLH1 and MSH2 mutations in yellow race and white race, and found that some mutations are particularly frequent in yellow race, which need to be taken into account for future personal medicine.

It is important to identify casual disease genes while combating diseases. Recently, linkage disequilibrium mapping is developed as one promising method to reveal the disease gene locations, thereby providing insights into disease 
mechanisms. Lin et al. ${ }^{9}$ proposed a new algorithm for linkage disequilibrium mapping, which demonstrates good performance on real datasets. On the other hand, differentially expressed genes can also give clues to genes related to phenotype change. Tang et al. ${ }^{10}$ developed a new algorithm for cancer classification based on gene expression data. The algorithm was confirmed to be effective on the test cases.

\section{Transcriptome Analysis}

Transcriptome data have been widely used in gene function prediction, disease subtype identification, compound analysis, and so on. In past years, most of traditional transcriptome data are generated with microarray chips, which can be used to monitor the expression of tens of thousands of genes simultaneously. However, it is challenging to mine useful signals out of the huge expression dataset due to the inherited noises. In this issue, Yu et al. ${ }^{11}$ proposed a new method to identify the differentially expressed (DE) genes in multi-conditional microarray datasets, and the identified DE genes are helpful for understanding biological processes that underlie phenotype changes.

Recently, a newly developed RNA-seq technology is attracting much attention in the area of transcriptome study due to its high resolution. Nevertheless, it is not trivial to estimate the transcript abundance levels from RNA-seq. Wang et al. ${ }^{12}$ compared the two popular techniques for estimating gene expression levels while handling RNA-seq data, and found that isoform-based strategy generally outperforms UI-based one. This study provides a clear guideline for scientists to choose appropriate techniques on analyzing RNA-seq data.

\section{Acknowledgments}

We would like to thank all reviewers for their invaluable contributions to the peer review process that have made this special issue possible. We also would like to thank Yixiang Shi of Shanghai Center for Bioinformatics Technology for his help in communications with the authors and reviewers.

\section{References}

1. Parasuram R, Lee JS, Yin PC, Somarowthu S, Ondrechen MJ, Functional classification of protein 3D structures from predicted local interaction sites, J Bioinform Comput Biol 8(Suppl 1):1-15, 2010.

2. Shih Th, Chen CM, Wang HW, Pai TW, Chang HT, BiMFG: Bioinformatics tools for marine and freshwater species, J Bioinform Comput Biol 8(Suppl 1):17-32, 2010.

3. Li Y, Jin K, Zhu Z, Yang J, Stepwise origin and functional diversification of the AFL subfamily B3 genes during land plant evolution, J Bioinform Comput Biol 8(Suppl 1):33-45, 2010.

4. Yu L, Gao L, Li k, A method based on local density and random walks for complexes detection in protein interaction networks, J Bioinform Comput Biol 8(Suppl 1):47-62, 2010 . 
5. Brown JB, Urata T, Tamura T, Arai MA, Bata TK, Akutsu T, Compound analysis via graph kernels incorporating chirality, J Bioinform Comput Biol 8(Suppl 1):63-81, 2010.

6. Tohsato Y, Baba T, Mazaki Y, Ito M, Ondrechen MJ, Environmental dependency of gene knockouts on phenotype microarray analysis in Escherichia coli, J Bioinform Comput Biol 8(Suppl 1):83-99, 2010.

7. Zhao X, Li ZF, Zhang XY, G6PD-MUTDB: A mutation and phenotype database of glucose-6-phosphate (G6PD) deficiency, J Bioinform Comput Biol 8(Suppl 1):101109, 2010.

8. Wei WQ, Chen J, Jiang F, Liu FQ, Fan R, Cheng Z, Shen M, Xue CY, Xu Y, Nan P, Liu L, Racial differences in MLH1 and MSH2 mutation: An analysis of yellow race and white race based on the InSiGH database, J Bioinform Comput Biol 8(Suppl 1):111$125,2010$.

9. Lin L, Wong L, Leong TY, Lai PS, Efficient mining of haplotype patterns for linkage disequilibrium mapping, J Bioinform Comput Biol 8(Suppl 1):127-146, 2010.

10. Tang KL, Yao Wj, Li TH, Li YX, Cao ZW, Cancer classification from the gene expression profiles by discriminant kernel-pls, J Bioinform Comput Biol 8(Suppl 1):147-160, 2010 .

11. Yu H, Tu K, Xie L, Li YY, DIGOUT: Viewing differential expression genes as outliers, J Bioinform Comput Biol 8(Suppl 1):161-175, 2010.

12. Wang $\mathrm{X}, \mathrm{Wu} \mathrm{ZP}$, Zhang XG, Isoform abundance inference provides a more accurate estimation of gene expression levels in RNA-seq, J Bioinform Comput Biol 8(Suppl 1):177-192, 2010. 